\title{
Correlações e análise de trilha de caracteres tecnológicos e a produtividade de fibra de algodão
}

\author{
Eulália Soler Sobreira Hoogerheide ${ }^{(1)}$, Roland Vencovsky ${ }^{(1)}$, Francisco José Correia Farias ${ }^{(2)}$, \\ Elêusio Curvêlo Freire ${ }^{(2)}$ e Eurípedes Maximiano Arantes ${ }^{(3)}$
}

\begin{abstract}
(1)Escola Superior de Agricultura Luiz de Queiroz, Dep. de Genética e Melhoramento de Plantas, Caixa Postal 83, CEP $13400-970$ Piracicaba SP. E-mail: essobrei@esalq.usp.br, rvencovs@esalq.usp.br (2)Embrapa Algodão, Núcleo Mato Grosso, Av. Campo Grande, no 612, sala 1, Centro, CEP 78850-000 Primavera do Leste, MT. E-mail: farias@cnpa.embrapa.br, eleusiofreire@hotmail.com ${ }^{(3)}$ Empaer/Universidade do Estado de Mato Grosso, Av. São João, s/no, Cavalhada, CEP 78200-000 Cáceres, MT. E-mail: agro.cac@unemat.br
\end{abstract}

\begin{abstract}
Resumo - O objetivo deste trabalho foi avaliar as relações entre os caracteres tecnológicos (variáveis independentes explicativas) e a produtividade de fibra do algodão (variável dependente) e identificar seus efeitos diretos e indiretos pela análise de trilha. Os caracteres tecnológicos foram: finura, resistência, comprimento, uniformidade de comprimento, alongamento e índice de fiabilidade do fio. Avaliaram-se 12 cultivares de algodoeiro herbáceo, no Estado do Mato Grosso, no ano agrícola 2000/2001. Estimaram-se as correlações, e fez-se a análise de trilha pelo método proposto por Wright. O caráter finura apresenta o maior efeito direto sobre a produtividade da fibra. A resistência, o comprimento e o alongamento têm efeito secundário. A uniformidade de comprimento e a fiabilidade apresentaram efeito indireto via finura. Um esquema de seleção restrito é necessário, para se eliminarem os efeitos indiretos negativos do índice de fiabilidade sobre a finura.
\end{abstract}

Termos para indexação: Gossypium hirsutum, seleção, finura.

\section{Correlations and path analysis of technological properties and the lint yield of cotton}

\begin{abstract}
The purpose of this work was to study the relations between technological properties (independent variables) and the lint yield of cotton (dependent variable). The technological properties analyze were: micronaire, strength, length, uniformity of length, elongation and index of thread spinning. Twelve cotton cultivars were evaluated, in Mato Grosso State, during the 2000/2001 crop season. Correlations and other parameters for path analysis were estimated, according to Wright. The trait micronaire showed the highest direct effect on lint yield; in addition, strength, length and elongation were secondary effects. The uniformity of length and the spinning inder showed indirect effect on micronaire. A restricted selection is necessary to eliminate the negative indirect effect of the spinning index on micronaire.
\end{abstract}

Index terms: Gossypium hirsutum, selection, micronaire.

\section{Introdução}

$\mathrm{O}$ algodoeiro produz uma das mais importantes fibras têxteis do mundo, pois oferece variados produtos de utilidade com grande relevância na economia brasileira e mundial, razão que a faz ser considerada uma das plantas de mais completo aproveitamento, figurando entre as dez maiores fontes de riqueza do agronegócio do Brasil (Costa et al., 2005).

Com o crescimento da produção nacional, a exportação é vista como solução para manutenção da rentabilidade da atividade a curto e médio prazo. Para isso, o algodão brasileiro deverá ser igualmente competitivo com o produzido em países exportadores, entre os quais se inclui a Austrália, cuja fibra é de excelente qualidade, motivo pelo qual a qualidade da fibra passa a ser uma exigência do agronegócio globalizado (Costa et al., 2005).

Um dos objetivos básicos dos programas de melhoramento do algodoeiro é obter genótipos que produzam mais fibra, com a qualidade requerida pelas indústrias têxteis. Porém, a produtividade é um caráter complexo e resultante da expressão e associação de diferentes componentes (Carvalho et al., 2002). O conhecimento do grau dessa associação, por meio de estudos de correlações, possibilita identificar caracteres 
que possam ser usados como critérios de seleção indireta para a produtividade. $\mathrm{O}$ conhecimento das correlações entre caracteres do algodão permite medir a magnitude da relação entre vários caracteres da planta e determina o caráter, no qual a seleção pode ser baseada, para o melhoramento em produtividade, precocidade e qualidade da fibra (Iqbal et al., 2003).

Com o objetivo de compreender melhor as causas envolvidas nas associações de caracteres, Wright (1921) propôs um método de desdobramento das correlações, estimadas em efeitos diretos e indiretos das variáveis sobre uma variável básica, denominado análise de trilha, o qual mede a influência direta de uma variável sobre a outra, que é independente das demais. A análise de trilha pode, portanto, ser feita a partir de correlações fenotípicas, genéticas ou ambientais (Cruz, 2001). Esta metodologia tem sido utilizada em várias espécies, entre elas o algodão (Tyagi et al., 1998; Iqbal et al., 2003).

Para que a avaliação da associação entre caracteres forneça estimativa e gere uma interpretação biologicamente correta, é necessário realizar-se a análise da multicolinearidade entre as variáveis independentes. Na presença de multicolinearidade moderada à severa, as variâncias associadas a certos estimadores, como por exemplo, dos coeficientes de trilha que medem efeitos diretos de variáveis explicativas sobre uma principal, podem atingir valores demasiadamente elevados, e são evidência de estimativas pouco confiáveis. Portanto, é necessário fazer sempre o diagnóstico da multicolinearidade dos dados, antes de se realizar o processamento da análise (Cruz \& Carneiro, 2003).

O objetivo deste trabalho foi avaliar as relações entre os caracteres tecnológicos e a produtividade da fibra do algodão e identificar seus efeitos diretos e indiretos, pela análise de trilha, de modo a auxiliar no processo de seleção.

\section{Material e Métodos}

Foram analisados dados obtidos do experimento conduzido no Município de São José dos Quatro Marcos, no Estado do Mato Grosso (15 $37^{\prime} \mathrm{S}, 58^{\circ} 10^{\prime} \mathrm{W} ; 230 \mathrm{~m}$ de altitude), no ano agrícola 2000/2001, pela Embrapa Algodão.

Avaliaram-se 12 cultivares de algodoeiro herbáceo: BRS Aroeira; BRS 201; BRS Cedro; Delta Opal; DP 4049; IAC 97-86; IPR 94; IPR 96; Epamig Liça;
FMT Saturno; BRS Camaçari; CNPA ITA 90, em delineamento de blocos ao acaso, com quatro repetições. A parcela experimental foi constituída de quatro fileiras de $5 \mathrm{~m}$, com espaçamento de $0,90 \mathrm{~m}$ entre fileiras e dez plantas por metro. A área útil da parcela foi constituída das duas fileiras centrais.

Avaliaram-se a produtividade de fibra de algodão e os caracteres tecnológicos. A produtividade foi calculada pelo produto da produtividade da área útil da parcela (pluma mais caroço) pela porcentagem de fibra, convertida para um hectare. Para isso, a porcentagem de fibra, para cada parcela, foi calculada com uma amostra de 20 capulhos, obtida do terço médio das plantas da área útil, e determinada após o beneficiamento, pela relação entre o peso da pluma e o peso total da amostra (pluma mais caroço). Os caracteres tecnológicos da fibra foram analisados em aparelho HVI (High Volume Instruments), os quais foram: finura (IM); resistência (mm); comprimento ( $\mathrm{mm}$ ); uniformidade de comprimento (\%); alongamento (\%); e índice de fiabilidade (Costa et al., 2005).

Inicialmente foi realizada a análise de variância para cada caráter. As estimativas de correlações genotípicas $\left(r_{\mathrm{g}}\right)$, fenotípicas $\left(\mathrm{r}_{\mathrm{f}}\right)$ e ambientais $\left(\mathrm{r}_{\mathrm{a}}\right)$, entre os caracteres, foram obtidas conforme Mode \& Robinson (1959), testadas a 1 e $5 \%$ de probabilidade pelo teste $\mathrm{t}$, a $\mathrm{n}-2$ graus de liberdade, e determinadas para todas as combinações de caracteres, com o objetivo de se obterem informações sobre a natureza e intensidade entre eles.

Foi realizada a diagnose da multicolinearidade, e o grau de multicolinearidade da matriz singular $\mathrm{X}^{\prime} \mathrm{X}$ foi estabelecido com base no número de condição (NC), que é a relação entre o maior e o menor autovalor da matriz. Se $\mathrm{NC}<100$, a multicolinearidade é denominada fraca e não constitui problema para a análise; se $100 \leq \mathrm{NC}<1000$, a multicolinearidade é considerada de moderada à forte; e se $\mathrm{NC} \geq 1000$, é considerada severa (Cruz \& Carneiro, 2003).

Posteriormente, foi feito o desdobramento das correlações fenotípicas, em efeitos diretos e indiretos dos caracteres de qualidade da fibra (variável independente) sobre a produtividade da fibra (variável dependente), por meio do modelo de análise de trilha (Wright, 1921).

A diagnose da multicolinearidade e todas as outras análises deste trabalho foram efetuadas pelo programa Genes (Cruz, 2001). 


\section{Resultados e Discussão}

O resumo das análises de variância encontra-se na Tabela 1. Verifica-se que houve diferença significativa para cinco dos sete caracteres avaliados, o que revela variabilidade genética entre as cultivares. Os coeficientes de variação foram baixos, de 0,7 a 9,6\%.

$\mathrm{Na}$ Tabela 2, encontram-se as estimativas das correlações fenotípicas $\left(r_{f}\right)$, genéticas $\left(r_{g}\right)$ e ambientais $\left(\mathrm{r}_{\mathrm{a}}\right)$. Verificam-se diferenças em sinal com as correlações genotípicas e ambientais, o que indica que as causas de variação genética e de ambiente podem afetar os caracteres, por meio de diferentes mecanismos fisiológicos. As correlações genéticas foram superiores às fenotípicas e ambientais, para a maioria dos casos. Essa superioridade é comentada por Gonçalves et al. (1996) como resultante dos efeitos modificadores do ambiente na associação dos caracteres genéticos.

Kang et al. (1983) mencionam que quando as magnitudes das correlações não são semelhantes, as correlações genotípicas são intrinsecamente mais úteis do que as correlações fenotípicas, para decidir estratégias de seleção. Porém, caracteres genotípica, mas não fenotipicamente correlacionados podem não ter valor prático na seleção, porque a seleção é, geralmente, realizada com base no fenótipo (Shukla et al., 1998). Por isso, preferiu-se utilizar a correlação fenotípica para a análise de trilha, por ser esta a que o melhorista manipula.

Conforme observado na Tabela 2, os caracteres que apresentaram correlações significativas com a produtividade de fibra foram: finura $(0,77)$; uniformidade de comprimento $(0,58)$; e índice de fiabilidade $(-0,70)$. Depreende-se desses resultados que a produtividade de fibra encontra-se diretamente relacionada à finura e à uniformidade de comprimento. Com relação ao índice de fiabilidade, as estimativas revelaram correlação negativa, o que demonstra uma associação inversa entre ambos os caracteres.

Tabela 1. Resumo das análises de variância para os caracteres: produtividade de fibra (Pfibra), finura, resistência (Res), comprimento (Comp), uniformidade de comprimento (Unf), alongamento (Alg) e índice de fiabilidade (Inf).

\begin{tabular}{|c|c|c|c|c|c|c|c|c|}
\hline Fonte de variação & GL & Pfibra & Finura & Res & Comp & Unf & Alg & Inf \\
\hline Bloco & 3 & $21.873,82$ & 0,02 & 1,79 & 1,02 & 2,24 & 0,99 & $2.090,14$ \\
\hline Cultivares & 11 & $161.721,65^{* *}$ & $0,74 * *$ & $18,87 * *$ & $1,11^{*}$ & $0,84^{\mathrm{ns}}$ & $0,76^{\mathrm{ns}}$ & $11.300,95 * *$ \\
\hline Resíduo & 33 & $18.507,37$ & 0,07 & 4,12 & 0,47 & 0,44 & 0,45 & $3.497,91$ \\
\hline Média & & $1.412,03$ & 4,52 & 28,26 & 29,78 & 85,11 & 10,35 & $2.094,70$ \\
\hline CV $(\%)$ & & 9,6 & 5,8 & 7,2 & 2,3 & 0,7 & 6,5 & 2,8 \\
\hline
\end{tabular}

ns Não-significativo. * e **Significativo a 5 e $1 \%$ de probabilidade, respectivamente, pelo teste $\mathrm{F}$.

Tabela 2. Estimativas dos coeficientes de correlações fenotípica $\left(r_{f}\right)$, genotípica $\left(r_{g}\right)$ e ambiental $\left(r_{a}\right)$, entre os caracteres: produtividade de fibra (Pfibra), finura, resistência (Res), comprimento (Comp), uniformidade de comprimento (Unf), alongamento (Alg) e índice de fiabilidade (Inf).

\begin{tabular}{|c|c|c|c|c|c|c|c|}
\hline Caráter & Correlação & Finura & Res & Comp & Unf & $\mathrm{Alg}$ & Inf \\
\hline \multirow[t]{3}{*}{ Pfibra } & $\mathrm{r}_{\mathrm{f}}$ & $0,77 * *$ & $0,21^{\mathrm{ns}}$ & $0,40^{\text {ns }}$ & $0,58^{*}$ & $-0,38^{\mathrm{ns}}$ & $-0,70^{*}$ \\
\hline & $r_{g}$ & $0,81 * *$ & $0,30^{\mathrm{ns}}$ & $0,51^{\mathrm{ns}}$ & $0,84 * *$ & $0,69^{*}$ & $-0,81 * *$ \\
\hline & $r_{a}^{g}$ & $0,40^{\mathrm{ns}}$ & $-0,29^{\text {ns }}$ & $0,11^{\mathrm{ns}}$ & $0,15^{\mathrm{ns}}$ & $0,13^{\mathrm{ns}}$ & $-0,28^{\mathrm{ns}}$ \\
\hline \multirow[t]{3}{*}{ Finura } & $\mathrm{r}_{\mathrm{f}}$ & 1 & $0,13^{\text {ns }}$ & $0,57 *$ & $0,74 * *$ & $-0,10^{\mathrm{ns}}$ & $-0,80 * *$ \\
\hline & $r_{g}$ & 1 & $0,26^{\mathrm{ns}}$ & $0,79 * *$ & $1,10^{* *}$ & $-0,15^{\mathrm{ns}}$ & $-0,85 *$ \\
\hline & $r_{a}^{g}$ & 1 & $-0,65^{*}$ & $-0,31^{\mathrm{ns}}$ & $0,08^{\mathrm{ns}}$ & $-0,01^{\mathrm{ns}}$ & $-0,74 * *$ \\
\hline \multirow[t]{3}{*}{ Res } & $r_{f}$ & - & 1 & $0,31^{\mathrm{ns}}$ & $0,28^{\mathrm{ns}}$ & $-0,26^{\mathrm{ns}}$ & $0,25^{\mathrm{ns}}$ \\
\hline & $r_{g}$ & - & 1 & $0,39^{\text {ns }}$ & $0,57^{*}$ & $-0,64 *$ & $0,08^{\mathrm{ns}}$ \\
\hline & $\mathrm{r}_{\mathrm{a}}$ & - & 1 & $0,19^{\mathrm{ns}}$ & $-0,21^{\mathrm{ns}}$ & $0,29^{\text {ns }}$ & $0,76^{* *}$ \\
\hline \multirow[t]{3}{*}{ Comp } & $\mathrm{r}_{\mathrm{f}}$ & - & - & 1 & $0,70^{*}$ & $-0,31^{\mathrm{ns}}$ & $-0,20^{\mathrm{ns}}$ \\
\hline & $r_{g}$ & - & - & 1 & $1,09 * *$ & $-0,52^{\text {ns }}$ & $-0,61 *$ \\
\hline & $\mathrm{r}_{\mathrm{a}}$ & -- & - & 1 & $0,27^{\mathrm{ns}}$ & $-0,11^{\mathrm{ns}}$ & $0,50^{\text {ns }}$ \\
\hline \multirow[t]{3}{*}{$\overline{U n f}$} & $r_{f}$ & - & - & - & 1 & $-0,18^{\mathrm{ns}}$ & $-0,37^{\mathrm{ns}}$ \\
\hline & $\mathrm{r}_{\mathrm{g}}$ & - & - & - & 1 & $0,19^{\text {ns }}$ & $-0,78 * *$ \\
\hline & $\mathrm{r}_{\mathrm{a}}$ & - & - & - & 1 & $-0,47^{\mathrm{ns}}$ & $0,19^{\mathrm{ns}}$ \\
\hline \multirow[t]{3}{*}{$\overline{\mathrm{Alg}}$} & $\mathrm{r}_{\mathrm{f}}$ & - & - & - & - & 1 & $0,14^{\mathrm{ns}}$ \\
\hline & $\mathrm{r}_{\mathrm{g}}$ & - & - & - & - & 1 & $0,27^{\mathrm{ns}}$ \\
\hline & $r_{a}^{5}$ & - & - & - & - & 1 & $0,01^{\mathrm{ns}}$ \\
\hline
\end{tabular}

nsNão-significativo. * e **Significativo a 5 e $1 \%$ de probabilidade, respectivamente, pelo teste $\mathrm{t}$. 
Cruz \& Carneiro (2003) ressaltam que quando um caráter correlaciona-se positivamente com alguns e negativamente com outros, há a indicação de se ter um cuidado adicional, pois, ao selecionar-se um determinado caráter, podem-se provocar mudanças indesejáveis em outros. Tais correlações são indicação da dificuldade que poderá haver em selecionar materiais que venham a ter elevada produtividade de fibra, finura e uniformidade de comprimento com bom índice de fiabilidade.

Quanto aos demais caracteres (Tabela 2), as maiores correlações fenotípicas encontradas foram: finura $\mathrm{x}$ comprimento $(0,57)$; finura $\mathrm{x}$ uniformidade de comprimento $(0,74)$; finura $x$ índice de fiabilidade $(-0,80)$; comprimento $\mathrm{x}$ uniformidade de comprimento $(0,70)$. Todas foram significativas e, na sua maioria, positivas, exceto finura $x$ índice de fiabilidade.

O estudo de correlações é uma medida de associação e não permite tirar conclusões sobre o estudo da relação de causa e efeito. Por isso, procedeu-se à análise de trilha, que investiga a relação de causa e efeito. Silva et al. (2005) mencionam que esta análise proporciona um conhecimento detalhado das influências dos caracteres envolvidos, e justificam a existência de correlações positivas e negativas, de alta e baixa magnitude, entre os caracteres estudados.

Para obtenção dos efeitos diretos e indiretos da análise de trilha, é necessário que a matriz $X^{\prime} X$ seja bem condicionada. Problemas de multicolinearidade podem torná-la singular, e fazer, conseqüentemente, com que as estimativas de quadrados mínimos não sejam viáveis (Cruz, 2001). O diagnóstico da multicolinearidade da matriz de correlações fenotípicas indicou $\mathrm{NC}<100$, o que caracteriza multicolinearidade fraca e não se constitui em um problema para a análise de trilha.

Ao observar-se a análise de trilha na Tabela 3, notase que o caráter finura apresentou alta correlação positiva $(0,77)$ e efeito direto alto $(0,54)$, o que indica que a seleção truncada com este caráter pode proporcionar ganho satisfatório na produtividade de fibra. A resistência revelou baixa correlação positiva $(0,21)$, e baixo efeito direto com a produtividade de fibra $(0,14)$.

O comprimento apresentou correlação positiva $(0,40)$, mas não significativa estatisticamente, e efeito direto negativo $(-0,20)$. Isso indica que tal correlação foi causada pelos efeitos indiretos, e que o caráter finura foi o que apresentou maior contribuição via indireta $(0,30)$, com $75 \%$ do efeito direto do comprimento sobre a produtividade da fibra. Porém, como a associação entre os caracteres não foi elevada, provavelmente a seleção simultânea não levaria a êxito no ganho genético.

Quanto à uniformidade de comprimento, seu coeficiente de correlação foi positivo e alto $(0,58)$, mas seu efeito direto mostrou-se baixo $(0,15)$. O efeito indireto da finura foi o que mais contribuiu (aproximadamente 69\%), para que a correlação entre uniformidade de comprimento e a produtividade da fibra fosse elevada. Neste caso, a melhor estratégia, segundo

Tabela 3. Estimativas dos efeitos direto e indireto, que envolveram a variável principal dependente produtividade de fibra (Pfibra) e as independentes explicativas: finura, resistência (Res), comprimento (Comp), uniformidade de comprimento (Unf), alongamento (Alg) e índice de fiabilidade (Inf).

\begin{tabular}{|c|c|c|}
\hline Caráter & Efeitos de associação & Estimativa \\
\hline \multirow[t]{7}{*}{ Finura } & Direto sobre Pfibra & 0,54 \\
\hline & Indireto via Res & 0,02 \\
\hline & Indireto via Comp & $-0,11$ \\
\hline & Indireto via Unf & 0,11 \\
\hline & Indireto via $\mathrm{Alg}$ & 0,03 \\
\hline & Indireto via Inf & 0,19 \\
\hline & Total & 0,77 \\
\hline \multirow[t]{7}{*}{ Res } & Direto sobre Pfibra & 0,14 \\
\hline & Indireto via Finura & 0,07 \\
\hline & Indireto via Comp & $-0,06$ \\
\hline & Indireto via Unf & 0,41 \\
\hline & Indireto via $\mathrm{Alg}$ & 0,08 \\
\hline & Indireto via Inf & $-0,06$ \\
\hline & Total & 0,21 \\
\hline \multirow[t]{7}{*}{ Comp } & Efeito direto sobre Pfibra & $-0,20$ \\
\hline & Efeito indireto via Finura & 0,30 \\
\hline & Efeito indireto via Res & 0,05 \\
\hline & Efeito indireto via Unf & 0,10 \\
\hline & Efeito indireto via Alg & 0,09 \\
\hline & Efeito indireto via Inf & 0,05 \\
\hline & Total & 0,40 \\
\hline \multirow[t]{7}{*}{$\overline{\mathrm{Unf}}$} & Direto sobre Pfibra & 0,15 \\
\hline & Indireto via Finura & 0,40 \\
\hline & Indireto via Res & 0,04 \\
\hline & Indireto via Comp & $-0,14$ \\
\hline & Indireto via $\mathrm{Alg}$ & 0,05 \\
\hline & Indireto via Inf & 0,09 \\
\hline & Total & 0,58 \\
\hline \multirow[t]{7}{*}{$\overline{\mathrm{Alg}}$} & Direto sobre Pfibra & $-0,29$ \\
\hline & Indireto via Finura & $-0,05$ \\
\hline & Indireto via Res & $-0,04$ \\
\hline & Indireto via Comp & 0,06 \\
\hline & Indireto via Unf & $-0,03$ \\
\hline & Indireto via Inf & $-0,03$ \\
\hline & Total & $-0,38$ \\
\hline \multirow[t]{7}{*}{$\overline{\operatorname{Inf}}$} & Direto sobre Pfibra & $-0,24$ \\
\hline & Indireto via Finura & $-0,43$ \\
\hline & Indireto via Res & 0,04 \\
\hline & Indireto via Comp & 0,04 \\
\hline & Indireto via Unf & $-0,05$ \\
\hline & Indireto via $\mathrm{Alg}$ & $-0,04$ \\
\hline & Total & $-0,70$ \\
\hline Coeficiente de determinação & & 0,72 \\
\hline Efeito da variável residual & & 0,53 \\
\hline
\end{tabular}


Cruz \& Carneiro (2003), pode ser a seleção simultânea de caracteres, com ênfase naqueles cujos efeitos indiretos são significativos; ou seja, uma seleção indireta por meio da finura.

O caráter alongamento apresentou correlação $(-0,38)$ e efeito direto negativo $(-0,29)$ sobre a produtividade da fibra, mas não significativa.

$\mathrm{O}$ índice de fiabilidade teve alta correlação negativa $(-0,70)$, mas seu efeito direto sobre a produtividade da fibra foi baixo $(-0,24)$. Nesse caso, a finura foi o caráter que mais contribuiu via indireta (61\%) para tal correlação. Tal associação é indicação da necessidade de se aplicar um esquema de seleção restrito, a fim de se eliminarem os efeitos indiretos negativos do índice de fiabilidade sobre a finura.

De modo geral, os seis caracteres estudados diferiram em seu grau de influência direta sobre a produtividade da fibra. Entre eles, a finura foi o que teve efeito positivo e relativamente alto, o que indica a presença de causa e efeito. Esse caráter está relacionado a ganhos na produtividade da fibra. Os caracteres resistência, comprimento e alongamento podem ser considerados secundários na influência sobre a produtividade da fibra. A uniformidade de comprimento e o índice de fiabilidade, apesar do efeito direto baixo, têm certamente efeitos indiretos não desprezíveis (positivos para uniformidade e negativos para fiabilidade), detectados ambos pela finura.

A presença de efeitos indiretos negativos mostra a dificuldade que se tem em selecionar apenas com base no comportamento da variável principal. Vencosvsky \& Barriga (1992) relatam que, aparentemente, ainda não se tem um método adequado para maximizar a resposta à seleção e considerar apenas os componentes principais da variável principal. Portanto, quando o processo de seleção for baseado apenas em produtividade da fibra, haverá perda do controle sobre o comportamento e o equilíbrio harmônico que devem existir entre seus componentes, requisito básico que caracteriza uma boa cultivar de algodoeiro.

\section{Conclusões}

1. Os caracteres tecnológicos têm influência sobre a produtividade de fibra de algodão.

2. O caráter finura apresenta efeito direto sobre a produtividade da fibra.

3. O índice de fiabilidade possui efeitos indiretos negativos sobre a finura.

\section{Agradecimentos}

Ao $\mathrm{CNPq}$, pela concessão de bolsa; à Embrapa Algodão, pela cessão dos dados.

\section{Referências}

CARVALHO, C.G.P. de; ARIAS, C.A.A.; TOLEDO, J.F.F. de; OLIVEIRA, M.F. de; VELLO, N.A. Correlações e análise de trilha em linhagens de soja semeadas em diferentes épocas. Pesquisa Agropecuária Brasileira, v.37, p.311-320, 2002.

COSTA, J.N. da; ALMEIDA, F. de A.C.; SANTANA, J.C.F. de; COSTA, I.L.L. da; WANDERELY, M.J.R.; SANTANA, J.C. da S. Técnicas de colheita, processamento e armazenamento do algodão. Campina Grande: Embrapa Algodão, 2005. 14p. (Circular técnica).

CRUZ, C.D. Programa GENES: aplicativo computacional em genética e estatística. Viçosa: UFV, 2001. 648p.

CRUZ, C.D.; CARNEIRO, P.C.S. Modelos biométricos aplicados ao melhoramento genético. Viçosa: UFV, 2003. 585p.

GONÇALVES, P.S.; MARTINS, A.L.M.; BORTOLLETO, N.; TANZIZI, M.R. Estimates of genetic parameters and correlations of juvenile characters based on open pollinated progenies of Hevea. Brazilian Journal of Genetics, v.19, p.105-111, 1996.

IQBAL, M.; CHANG, M.A.; IQBAL, M.Z. Correlation and path coefficient analysis of earliness and agronomic characters of upland cotton in Multan. Pakistan Journal of Agronomy, v.2, p.160-168, 2003.

KANG, M.S.; MILLER, J.D.; TAI, P.Y.P. Genetic and phenotypic path analyses and heritability in sugarcane. Crop Science, v.23, p.643-647, 1983.

MODE, J.C.; ROBINSON, H.F. Pleiotropism and genetic variance and covariance. Biometrics, v.15, p.518-537, 1959.

SHUKLA, S.; SINGH, K.; PUSHPENDRA. Correlation and path coefficient analysis of yield and its components in soybean (Glycine $\max ($ L.) Merrill). Soybean Genetics Newsletter, v.25, p.67-70, 1998.

SILVA, S.A.; CARVALHO, F.I.F. de; NEDEL, J.L.; CRUZ, P.J.; SILVA, J.A.G. da; CAETANO, V. da R.; HARTWIG, I.; SOUSA, C. da S. Análise de trilha para os componentes de rendimento de grãos em trigo. Bragantia, v.64, p.191-196, 2005.

TYAGI, A.P.; MOR, B.R.; SINGH, D.P. Path analysis in upland cotton (G. hirsutum L.). Indian Journal of Agricultural Research, v.22, p.137-142, 1998.

VENCOVSKY, R.; BARRIGA, P. Genética biométrica no fitomelhoramento. Ribeirão Preto: RBG, 1992. 496p.

WRIGHT, S. Correlation and causation. Journal of Agricultural Research, v.20, p.557-585, 1921.

$\overline{\text { Recebido em } 24 \text { de maio de } 2007 \text { e aprovado em } 18 \text { de setembro de } 2007}$ 